\section{Home-Made Holey-Film Grids}

Perforated film can be used as test specimens for astigmatism compensation in EM lenses. They also make good specimens for first teaching students on how to focus and stigmate a TEM. The following procedure was adapted from Paul Elsner. These procedures give fewer perforations of a small size and with smooth edges.

1) Make a $0.4 \%$ Formvar solution in 1,2-dichlornethane $(0.1 \mathrm{gm} / 25 \mathrm{~mL})$

in a suitable vessel, such as a coplin jar.

2) Place Formvar solution into a chamber where the relative humidity can be set at about 70\%. Dip glass slides into Formvar solution.

3) Immediately after slides are dipped into Formvar, place them in a vessel (such as another coplin jar) containing blotter paper saturated with dichloroethane. Slides should not touch blotter paper. Leave slides in vapors for 2 minutes to allow Formvar solution to drain for making thin films, let stand in high humidity for holes to form. (Holes are formed in Formvar when slides are removed from vapors and exposed to the high humidity of chamber atmosphere.)

4) Allow slides to dry within moist chamber, then place in closed container. Mount grids on holey film the same way as for Formvar grids.

5) Before carbon coating, holes must be smoothed. Tape paper or parafllm with coated grids to inside cover of Petri dish. Place cover over Petri dish bottom containing 1:10 solution of chloroform:methanol for 1 minute.

6) Carbon coat using about half of the $0.04^{\prime \prime}$ diameter point of a carbon rod.

Steps 2 through 4 are carried out in the same moist environment, a box or oven (oft) with the necessary humid atmosphere.

\section{Polychrome Stain for Epoxy Sections}

This is a protocol we have used that gives wonderful results:

1. Blue Stain: Methylene blue $0.13 \mathrm{gm}$, Azure $\mid 10.02 \mathrm{gm}$, Glycerol $10 \mathrm{~mL}$, Methyl alcohol $10 \mathrm{~mL}$, distilled $\mathrm{H}_{2} \mathrm{O} 80 \mathrm{~mL}$ (stir and filter, keeps 6 months)

2. Red stain: Stock solution: Basic fuchsin $0.2 \mathrm{gm}$, distilled $\mathrm{H}_{2} \mathrm{O}$

$100 \mathrm{~mL}$ (stir and filter, keeps 6 months)

Working solution: dilute stock $1: 4$ in distilled $\mathrm{H}_{2} \mathrm{O}$ (make fresh daily)

3. Sodium Hydroxide $1 \%$, made fresh daily

Procedure:

1. Flood slide with blue stain $15-60$ seconds, depending on temperature and material.

2. Add 4-6 drops $\mathrm{NaCH}$ to the stain and mix by filting the slide, about 10 seconds total time.

3. Wash in running water and dry on hotplate. Blue stain can be destained by heating.

4. Add red stain for $15-30$ seconds on hotplate. (NOTE: Red stain cannot be destained)

5. Rinse with running water and dry.

Reterences:

Mackay and Mead. 1970. 26th EMSA meetings pp.296-297. Modi

fied by Griftin and Fahrenbach, Oregon Regional Primate Research Center

Linda M. Fox, Loyola University Medical School (Chicago)

Bruce Wagner, lowa State University

\section{VIDX Scan} Digital Imaging

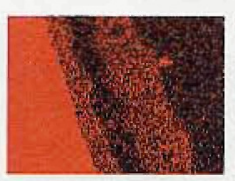

\author{
Digital Imaging \\ X-ray mapping \\ Image area: 16,000 by 16,000 \\ Gray levels: 65,536
}

